\title{
Biorthonormal Matrix-Product-State Analysis for Non-Hermitian Transfer-Matrix Renormalization-Group in the Thermodynamic Limit
}

\author{
Yu-Kun Huang ${ }^{*}$ \\ Graduate school of Engineering Science and Technology, Nan Jeon Institute \\ of Technology, Tainan 73746, Taiwan
}

\begin{abstract}
We give a thorough Biorthonormal Matrix-Product-State (BMPS) analysis of the Transfer-Matrix Renormalization-Group (TMRG) for non-Hermitian matrices in the thermodynamic limit. The BMPS is built on a dual series of reduced biorthonormal bases for the left and right Perron states of a non-Hermitian matrix. We propose two alternative infinite-size Biorthonormal TMRG (iBTMRG) algorithms and compare their numerical performance in both finite and infinite systems. We show that both iBTMRGs produce a dual infinite-BMPS (iBMPS) which are translationally invariant in the thermodynamic limit. We also develop an efficient wave function transformation of the iBTMRG, an analogy of McCulloch in the infinite-DMRG arXiv:0804.2509 (2008)], to predict the wave function as the lattice size is increased. The resulting iBMPS allows for probing bulk properties of the system in the thermodynamic limit without boundary effects and allows for reducing the computational cost to be independent of the lattice size, which are illustrated by calculating the magnetization as a function of the temperature and the critical spin-spin correlation in the thermodynamic limit for a 2D classical Ising model.
\end{abstract}

Keywords: Transfer-matrix renormalization-group, Density-matrix renormalizationgroup, Biorthonormal matrix-product-state, Correlation function.

PACS classification: 05.10.Cc; 05.50.+q; 02.70.-c; 05.70.-a

\section{Introduction}

It has been widely understood that the Matrix-Product-State (MPS) ansatz constitutes the basis of many numerical algorithms in computational physics, notably the Density-Matrix Renormalization-Group (DMRG) [1] and the Time-Evolving Block Decimation (TEBD) [2]. There are two main variants of the DMRG algorithm. The finite-size algorithm was by now

\footnotetext{
${ }^{*}$ Corresponding author. E-mail address: ykln@mail.njtc.edu.tw;
} 
realized to compute variationally the ground state of a strongly correlated 1D quantum system within the class of MPS [3]. While the infinite-size algorithm grows the system by adding iteratively one or more sites and reaches asymptotically, at the fixed point, a translationally invariant MPS state (invariant under translations of some fixed number of lattice sites), it was originally conceived as only for obtaining the initial MPS wave function for the finite-size algorithm. Until recently, interest in directly obtaining the translationally invariant MPS wave function in the thermodynamic limit was rekindled by the ideas of infinite-TEBD (iTEBD) of Vidal [4] and infinite-DMRG (iDMRG) of McCulloch [5]. Both approaches allow for probing bulk properties of the system in the thermodynamic limit without the influence of boundary conditions and the resulting infinite-MPS (iMPS) can reduce the computational cost of real-time simulations to be independent of the lattice size. The main difference between these two methods lies in the scheme for the local update of the tensors in the MPS. The iTEBD applies a single bond evolution operator to each site simultaneously which amounts to a power method while the iDMRG obtains the center matrix variationally by using a very efficient local eigensolver.

On the other hand, after nearly two decades of development, the application of DMRG methods has spread over a great variety of fields. One major branch of the DMRG is the Transfer-Matrix Renormalization-Group (TMRG) which can be applied to, e.g., the strongly correlated classical systems [6], the thermodynamics of 1D quantum systems at finite temperature [7], the stochastic transfer matrix of a cellular automaton [8], the nonequilibrium systems in statistical physics [9], and the general Markov random field in image modeling [10]. Different from the DMRG, the TMRG usually deals with non-Hermitian matrices which involve much more numerical demand in view of the existence of distinct left and right Perron states (i.e., the eigenstates associated with the maximum eigenvalue which we will refer to as the Perron root) of the transfer matrix. This leads to the selection of the reduced density matrix becoming ambiguous. Enss and Schollwöck [8] had provided a comparative discussion on several choices of the density matrix proposed in the literature for the non-Hermitian TMRG. Unfortunately, all these selections of density matrices cause the conventional TMRG fail to fit the framework of MPS analysis. In a recent paper [11], the author proposed a new TMRG algorithm called Biorthonormal Transfer-Matrix Renormalization-Group (BTMRG) which employed a dual set of biorthonormal bases to construct the renormalized transfer matrix and reduced the numerical complexity for nonHermitian matrices to be the same as the Hermitian case. Numerical simulations for a real non-Hermitian matrix showed that the BTMRG exhibits significant improvement on the efficiency and accuracy than conventional TMRG. Here, a dual biorthonormal bases indicate any two sets of vectors $\{|\alpha\rangle\}_{\alpha=1, \cdots, m}$ and $\{|\beta\rangle\}_{\beta=1, \cdots, m}$ that satisfy $\langle\alpha \mid \beta\rangle=\delta_{\alpha \beta}$. In the previous paper, the BTMRG was described in a traditional formulation. In this paper, we will show that the employment of the biorthonormal bases enables the BTMRG to be perfectly reformulated within the framework of MPS analysis where the two Perron states can be 
represented as a dual Biorthonormal Matrix-Product-States (BMPS). We will propose two alternative methods for building the BMPS. At the same time of doing so, two natural questions arise: Does the infinite-size variant of the BTMRG (iBTMRG) create asymptotically a dual infinite biorthonormal MPS (iBMPS) that are translationally invariant? Does there exist an efficient transformation in iBTMRG, just as the one in iDMRG [5], for predicting the wave function as the lattice size is increased? In this paper, both questions will be addressed confirmatively. Here, instead of the two-site iDMRG where the non-zero truncation of the wave function often leads to undesirable effects of the convergence of the iMPS [5], we will use the "single-site" iBTMRG scheme which achieves zero truncation similar to the single-site iDMRG. In addition, by using a special E•S•E configuration, the critical two-point correlation functions in the thermodynamic limit, especially when the distance between the two points approaches infinity, will be shown to be easily obtained.

The rest of this paper is organized as follows. In section 2, we present a brief review of the MPS analysis of the standard DMRG in finite systems and reformulate the BTMRG algorithm with the E•S•E configuration proposed in [11] entirely in terms of the BMPS language. In section 3, we propose two alternative methods to build the BMPS of the left and right Perron state for non-Hermitian matrices and develop an iBTMRG algorithm to obtain the asymptotics of the dual iBMPS wave functions that are translationally invariant. We also develop an efficient wave function transformation in iBTMRG for predicting the BMPS as the lattice size is increased. Section 4 compares the numerical performance of our iBTMRG algorithms in finite systems for a real non-Hermitian matrix of an anisotropic Ising model. The magnetization as a function of the temperature and the critical two-point correlation function of an isotropic Ising model in the thermodynamic limit are also plotted. Finally, in section 5, some conclusions are drawn.

\section{Biorthonormal MPS analysis of BTMRG in finite systems}

The connection between DMRG and MPS was first found by Östlund and Rommer [12] who identified the thermodynamic limit of DMRG with a position-independent matrix product wave function. The discovery of the MPS on which the DMRG operates has placed the algorithm on a firm footing, provided a deeper understanding, and allowed a concrete and easy to manipulate description of the DMRG. The standard DMRG and MPS formulation was established on a series of reduced orthonormal bases. However, when considering both left and right Perron states of non-Hermitian transfer matrices, the MPS must be built on a dual series of reduced biorthonormal bases which we will refer to as biorthonormal matrix-product states (BMPS). In this section, we briefly review the finite-size BTMRG algorithm proposed in [11] but reformulate it entirely in terms of a BMPS language. For further information about MPS, see [13]. 


\subsection{MPS formulation of DMRG in finite systems}

Let us start with the MPS formulation of the standard DMRG in finite systems. Throughout this paper, we focus on the MPS of open boundary conditions. A spin chain can be bipartitioned into two parts: the system block where the spins are labeled by $s_{i}$, and the environment block where the spins are labeled by $\varepsilon_{i}$. Then we denote an MPS on an L-site lattice by the form

$$
|\psi\rangle=\sum_{s_{i}, \varepsilon_{i}} A_{1}^{s_{1}} \cdots A_{p}^{s_{p}} \Lambda E_{q}^{\varepsilon_{q}} \cdots E_{1}^{\varepsilon_{1}}\left|s_{1} \cdots s_{p} \varepsilon_{q} \cdots \varepsilon_{1}\right\rangle
$$

where $p+q=L$ and each matrix at each site has dimension $m \times m$ with the exception that the end matrices $A_{1}^{s_{1}}$ and $E_{1}^{\varepsilon_{1}}$ are a row vector and a column vector respectively. The $A$-matrices ( $E$-matrices) satisfy the left (right)-normalization condition $\sum_{s_{i}} A_{i}^{s_{i} \dagger} A_{i}^{s_{i}}=I$ $\left(\sum_{\varepsilon_{i}} E_{i}^{\varepsilon_{i}} E_{i}^{\varepsilon_{i} \dagger}=I\right)$. It is very useful to express an MPS in a tensor network representation (see Schollwöck [13]) as in Fig. 1(a). For example, the set of matrices $A_{p}^{s_{p}}$ at the $p$-th site of the system block corresponds to a tensor $\left(A_{p}\right)_{\alpha_{p-1}, \alpha_{p}}^{s_{p}}$ with two bond indices $\alpha_{p-1}$ and $\alpha_{p}$ and one physical index $s_{p}$. The bond index labels the reduced orthonormal basis state of the system block at different lengths and the physical index labels the state of the spin. Thus the center matrix $\Lambda$ represents in fact the wave function of the superblock in the reduced orthonormal basis $\left\{\left|\alpha_{p} \xi_{q}\right\rangle\right\}_{\alpha, \xi=1, \cdots, m}$ (see Fig. 1(a)). The essential notion of the MPS formulation of the finite-size variant of DMRG is that of local updates; that is, we free a matrix, say $E_{q}^{s_{q}}$, at a time while keeping the others fixed, update it by $A_{p+1}^{s_{p+1}}$ through an efficient optimization of the total energy, and shift the center matrix to the right by one site. When all matrices in the MPS are updated once, it is called a sweep. Repeat the sweep until convergence is reached.

\subsection{E•S•E scheme and the related BMPS representation}

Now consider a special bi-partitioning of the chain as indicated by the notation E•S•E where the system block is taken as a consecutive segment of sites around the center of the chain and the environment block is taken as two equal separate segments surrounding the system block. The superblock is updated at a time by freeing (or adding) two spins in between the two sub-systems so that the matrices of the MPS must be associated with two distant sites. This special configuration is shown to be particularly adapted to the calculation of two-point correlation functions of $1 \mathrm{D}$ quantum systems or $2 \mathrm{D}$ classical lattice

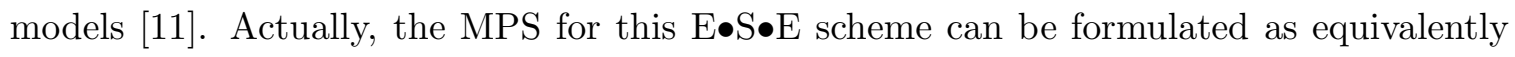
as the MPS for the standard DMRG. By folding the spin chain from the center so that the two sub-systems and the two free spins are aligned, every two aligned spins can be regarded as a single big spin and the resulting new chain has the same configuration as the more 
standard S•E scheme. To avoid the notation from getting too involved, it is convenient and comprehensive to represent this MPS as a tensor network as in Fig. 1(b) where the tensor $\left(A_{p}\right)_{\alpha_{p-1}, \alpha_{p}}^{\vdots}$, now, with two physical indices representing the states of the two spins. Alternatively, following McCulloch [5], such an MPS can be simply expressed as

$$
|\psi\rangle=A_{1}^{\vdots} \cdots A_{p}^{\vdots} \Lambda E_{q}^{:} \cdots E_{1}^{:}
$$

In this notation, although the ket basis vectors of the MPS are suppressed and the alphabets labeling the state of the aligned spin pairs are simply denoted by two dots, all information are preserved. It is easy to understand that, here, the MPS matrices are local state valued albeit not explicitly written.

When a non-Hermitian transfer matrix $T$ is considered, the left and right Perron state are generally distinct. Suppose the left $|\psi\rangle$ and right $|\varphi\rangle$ Perron state have MPS as follows.

$$
\left\{\begin{array}{l}
|\psi\rangle=\bar{A}_{1}^{:} \cdots \bar{A}_{p}^{:} \overline{\Lambda E}_{q} \cdots \bar{E}_{1}^{:} \\
|\varphi\rangle=\bar{B}_{1}^{:} \cdots \bar{B}_{p}^{\vdots} \overline{\Gamma F_{q}} \cdots \bar{F}_{1}^{\vdots}
\end{array}\right.
$$

When we free the two spins associated with the matrices $\bar{E}_{q}^{:}$and $\bar{F}_{q}^{:}$, we are actually carrying out the local updating by maximizing the quantity $\langle\psi|T| \varphi\rangle-\lambda\langle\psi \mid \varphi\rangle$. Hence, if we impose the so-called left- and right-biorthonormal conditions on the MPS matrices

$$
\sum_{:} \bar{B}_{i}^{: \dagger} \bar{A}_{i}^{:}=\sum_{:} \bar{E}_{i}^{:} \bar{F}_{i}^{\dagger \dagger}=I
$$

then the optimization will be equivalent to an eigenvalue problem of the reduced transfer matrix $\bar{T}$ that can be expressed as a tensor network as in Fig. 2 (see Schollwöck [13]) where the transfer matrix $T$ is expressed as a Matrix-Product-Operator (MPO) and $\sigma_{L}$ and $\sigma_{R}$ label the states of the free distant spin pair associated with the matrices $\bar{E}_{q}^{\text {: }}$ and $\bar{F}_{q}$. The matrix elements of the reduced transfer matrix can be written explicitly as $\bar{T}_{\alpha_{p} \sigma_{L} \sigma_{R} \xi_{q-1}, \beta_{p} \sigma_{L}^{\prime} \sigma_{R}^{\prime} \zeta_{q-1}}=\left\langle\alpha_{p} \sigma_{L} \sigma_{R} \xi_{q-1}|T| \beta_{p} \sigma_{L}^{\prime} \sigma_{R}^{\prime} \zeta_{q-1}\right\rangle$. If the pair of MPS states Eq. (3) satisfies the biorthonormal conditions Eq. (4), we refer to the MPS as a dual BMPS. Thus, the BTMRG algorithm can be perfectly fitted into the framework of MPS analysis and enjoy the same numerical complexity as the Hermitian case in DMRG algorithm. In this paper, we will propose two alternative methods for determining the BMPS for the Perron state and compare their numerical performance. The details will be described in section 3 .

\subsection{Density matrix and canonical form of BMPS representations}

Unlike the Hermitian Hamiltonian in DMRG that there is a unique normalized ground state, there are two distinct Perron states in TMRG so that the density operator for the Perron state of the system must be taken as $\widehat{\rho}=|\varphi\rangle\langle\psi|$ (with proper normalization $\langle\psi \mid \varphi\rangle=1$ ). Thus, from Eq. (3), we can readily obtain the reduced density operator for the system and environment block as 


$$
\left\{\begin{array}{c}
\widehat{\rho}_{S}=\sum_{\beta_{p}, \alpha_{p}}\left(\overline{\Gamma \Lambda}^{\dagger}\right)_{\beta_{p}, \alpha_{p}}\left|\beta_{p}\right\rangle\left\langle\alpha_{p}\right| \\
\widehat{\rho}_{E}=\sum_{\zeta_{q}, \xi_{q}}\left(\bar{\Gamma}^{\dagger} \bar{\Lambda}\right)_{\zeta_{q}, \xi_{q}}\left|\zeta_{q}\right\rangle\left\langle\xi_{q}\right|
\end{array}\right.
$$

Note that $\left\langle\alpha_{p} \mid \beta_{p}\right\rangle=\delta_{\alpha \beta}$ and $\left\langle\xi_{q} \mid \zeta_{q}\right\rangle=\delta_{\xi \zeta}$ in view of the biorthonormal conditions. However, given a BMPS as in Eq. (3), we can always construct another BMPS by introducing an arbitrary invertible (non-unitary) transformation to the matrices $\bar{E}_{q}^{:}$and $\bar{F}_{q}^{\text {: }}$ (i.e., applying a non-unitary basis transformation $X$ to the current biorthonormal bases $\left\{\left|\xi_{q}\right\rangle\right\}$ and $\left.\left\{\left|\zeta_{q}\right\rangle\right\}\right)$ such that ${\overline{E^{\prime}}}_{q}^{:}=X^{-1} \bar{E}_{q}^{:}$and ${\overline{F^{\prime}}}_{q}^{:}=X^{\dagger} \bar{F}_{q}^{:}$remain right-biorthonormal. Similar results ${\overline{A^{\prime}}}_{p}^{:}=\bar{A}_{p}^{:} Y^{-1 \dagger}$ and ${\overline{B^{\prime}}}_{p}^{:}=\bar{B}_{p}^{:} Y$ are obtained by applying a transformation $Y$ to the current biorthonormal bases $\left\{\left|\alpha_{p}\right\rangle\right\}$ and $\left\{\left|\beta_{p}\right\rangle\right\}$. Thus the varied BMPS turns out to be

$$
\left\{\begin{array}{l}
|\psi\rangle=\bar{A}_{1} \cdots{\overline{A^{\prime}}}_{p}^{:} \overline{\Lambda^{\prime} E_{q}^{\prime}} \cdots \bar{E}_{1}^{:} \\
|\varphi\rangle=\bar{B}_{1}^{\vdots} \cdots \overline{B_{p}^{\prime}}: \overline{\Gamma^{\prime} F_{q}^{\prime}}: \cdots \overline{F_{1}}
\end{array}\right.
$$

where the new center matrices become $\overline{\Lambda^{\prime}}=Y^{\dagger} \bar{\Lambda} X$ and $\overline{\Gamma^{\prime}}=Y^{-1} \bar{\Gamma} X^{-1 \dagger}$ and the new reduced density operators are simply a similar transform of the old density operators: $\widehat{\rho}_{S}^{\prime}=$ $Y^{-1} \widehat{\rho}_{S} Y$ and $\widehat{\rho}_{E}^{\prime}=X^{-1} \widehat{\rho}_{E} X$. This implies that if we employ a suitable transformation, we can canonize the form of the BMPS representation.

Given the BMPS in Eq. (3), assume the density matrix $\rho_{S}=\overline{\Gamma \Lambda}^{\dagger}=Y D Y^{-1}$ is diagonalizable, then it can be readily obtained that $\rho_{E}=\bar{\Gamma}^{\dagger} \bar{\Lambda}=X D X^{-1}$ where $X=$ $\bar{\Gamma}^{\dagger} Y^{-1 \dagger}$. Since $X$ and $Y$ are unique up to a scaling, we can replace $X$ and $Y$ by $X \lambda_{1}$ and $Y \lambda_{2}$ where $\lambda_{1}$ and $\lambda_{2}$ are two diagonal matrices. Accordingly, we have new center matrices $\overline{\Lambda^{\prime}}=\lambda_{2} D \lambda_{1}=\sqrt{D}$ and $\overline{\Gamma^{\prime}}=\lambda_{2}^{-1} \lambda_{1}^{-1}=\sqrt{D}$ by choosing $\lambda_{2}=D^{-1 / 2} \lambda_{1}^{-1}$. Thus the canonical form of BMPS reads

$$
\left\{\begin{array}{l}
|\psi\rangle=\bar{A}_{1}^{\vdots} \cdots \bar{A}_{p}^{\vdots} \sqrt{D E_{q}}: \cdots \bar{E}_{1}^{:} \\
|\varphi\rangle=\bar{B}_{1}^{\vdots} \cdots \bar{B}_{p}^{\vdots} \sqrt{D \bar{F}_{q}} \cdots \bar{F}_{1}^{\vdots}
\end{array}\right.
$$

and the density matrix bears the canonical form $\rho_{S}=\rho_{E}=D$. Surprisingly, according to our practical simulation, for large enough system size, $\rho_{S}=Y D Y^{-1}$ always exits and the eigenvalues are always real non-negative.

\section{Biorthonormal MPS analysis of iBTMRG in the thermo- dynamic limit}

The iDMRG grows the system at a time by adding one or two sites at the center of the lattice and produce a fixed point of iMPS that is translationally invariant. By thinking

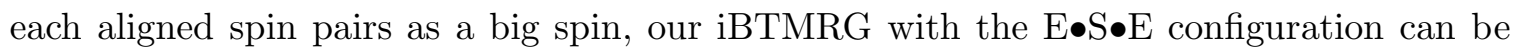


formulated as equivalently as the iDMRG. Throughout this paper, we choose the "singlesite" iBTMRG algorithm based on the following reasons. First, "two-site" iBTMRG means adding four spins at a time where the dimension of the reduced transfer matrix significantly increases. Second, the inevitable non-zero truncation of the wave function of the twosite iBTMRG often leads to a less-well converged wave function [5]. Third, the single-site iBTMRG can achieve zero truncation of the wave function and the problem of being trapped in local minimum can be avoided by introducing White's correction [14] to the reduced biorthonormal bases.

In this paper, many formulations are derived from a common basic procedure which we will refer to as the Biorthonormalization Procedure. Given two arbitrary bases $\{|\alpha\rangle\}_{\alpha=1, \cdots, m}$ and $\{|\beta\rangle\}_{\beta=1, \cdots, m}$, suppose $A \equiv\left[|\alpha\rangle_{\alpha=1, \cdots, m}\right]$ and $B \equiv\left[|\beta\rangle_{\beta=1, \cdots, m}\right]$ are two matrices formed with $|\alpha\rangle$ and $|\beta\rangle$ as their columns respectively. By carrying out the SingularValue-Decomposition (SVD) $A^{\dagger} B \equiv U \Sigma V^{\dagger}$, we can readily obtain $(A P)^{\dagger}(B W)=I$ where $P=U \Sigma^{-1 / 2}$ and $W=V \Sigma^{-1 / 2}$. This means that, by applying the non-unitary basis transformation $P$ and $W$ to the original bases $\{|\alpha\rangle\}$ and $\{|\beta\rangle\}$, we can obtain a dual biorthonormal bases $A P$ and $B W$.

\subsection{Two alternative iBTMRG algorithms in the thermodynamic limit}

We first propose an iBTMRG algorithm where the main steps are depicted in Fig. 3. The iBTMRG starts with an initialization procedure (i.e., $n=1$ ) which selects a minimum length of the lattice (depends on the number $m$ of states kept by BTMRG), obtains the respective reduced bases of the two sub-systems from the Perron states, and proceeds to biorthonormalize them. The routine in Fig. 3 grows the lattice by adding first two sites to the system block and then adding two sites to the environment block, which constitute a period of the whole algorithm with respect to a repeated fragment of the final iBMPS. At each time when adding new sites to the lattice, we obtain the Perron states (e.g., $\psi^{*}$ ) in the reduced biorthornormal bases, carry out an SVD for the Perron states to obtain the reduced bases of the enlarged block (e.g., $\psi^{:}=A_{n+1}^{:} \Lambda_{n+1}$ where $\sum A_{n+1}^{: \dagger} A_{n+1}^{:}=I$ ), and biorthonormalize them to build a new biorthonormal bases of the enlarged block (e.g., the right bond states of the tensors $\bar{A}_{n+1}^{:}$and $\bar{B}_{n+1}^{:}$). If the lattice size grows large enough, we can additionally canonize the BMPS. This step is convenient for the purpose of checking the convergence of the algorithm. According to the previous description, the biorthonormalization and the canonization procedures are simply to apply two successive non-unitary basis transformation to the bases obtained from the SVD, we can combine these transformation together and denote them by $P, W, Q$, and $R$ with respect to $A, B, E$, and $F$ matrices respectively (e.g., $\bar{A}_{n+1}^{:}=A_{n+1}^{:} P_{n+1}$ and $\bar{E}_{n+1}^{:}=Q_{n+1} E_{n+1}^{:}$, and thus $\left.\bar{\Lambda}_{n+1}=P_{n+1}^{-1} \Lambda_{n+1} Q_{n+1}^{-1}\right)$. Note that, during the SVD in step 2, we keep zero truncation of the wave function when obtaining the reduced basis of the enlarged block. The resulting BMPS read 


$$
\left\{\begin{array}{l}
|\psi\rangle=\bar{A}_{1}^{:} \cdots \bar{A}_{n}^{:} \bar{\Lambda}_{n} \bar{E}_{n}^{:} \cdots \bar{E}_{1}^{:} \\
|\varphi\rangle=\bar{B}_{1}^{:} \cdots \bar{B}_{n}^{:} \bar{\Gamma}_{n} \bar{F}_{n}^{:} \cdots \bar{F}_{1}^{:}
\end{array}\right.
$$

The above algorithm builds the biorthonormalized bases from the reduced bases derived from the SVD that are based on another biorthonormal bases. One may notice that there exist actually a lot of possibilities to build the biorthonormal bases. Nevertheless, the process of SVD is critical. We can also jump the SVD and directly biorthonormalize $\psi$ : and $\varphi^{:}$. But such a numerical procedure will become unstable very quickly. Here, we provide another algorithm which is very different from the above algorithm in nature. The alternative algorithm is depicted in Fig. 3 with the steps 2-3 enclosed in a rectangle being replaced by the routine depicted in Fig. 4. To the contrary, this algorithm constructs the biorthonormalized bases from the reduced bases derived from the SVD that are based on two orthonormal bases. The first major change lies in step 2 where before performing SVD the Perron states must be restored to be in terms of their own orthonormal bases, e.g., $\psi^{\prime:}=$ $P_{n} \psi: Q_{n}$. The second change lies in step 3 where before performing the biorthonormalization procedure the reduced bases derived from the SVD must be transformed back to be in terms of the previous biorthonormal bases, e.g., $A_{n+1}^{\prime:}=P_{n}^{-1} A_{n+1}^{:}$. As it turns out, two remarkable properties emerge, e.g., $\bar{A}_{n+1}^{:}=P_{n}^{-1} A_{n+1}^{:} P_{n+1}$ and $\bar{A}_{1} \cdots \bar{A}_{n}^{:}=A_{1} \cdots A_{n}^{:} P_{n+1}$. Similar relation holds for the $B, E$, and $F$ matrices. Accordingly, the BMPS can also be expressed in terms of two series of reduced orthonormal bases.

$$
\left\{\begin{aligned}
|\psi\rangle & =A_{1} \cdots A_{n}^{\vdots} \Lambda_{n} E_{n}^{:} \cdots E_{1} \\
|\varphi\rangle & =B_{1}^{\vdots} \cdots B_{n}^{\vdots} \Gamma_{n} F_{n}^{\vdots} \cdots F_{1}
\end{aligned}\right.
$$

where $\Lambda_{n}=P_{n} \bar{\Lambda}_{n} Q_{n}$ and $\Gamma_{n}=W_{n} \bar{\Gamma}_{n} R_{n}$. For convenience, in this paper, the former algorithm will be referred to as iBTMRG A and the latter iBTMRG B.

\subsection{Efficient wave function prediction and the fixed point of the iBMPS}

In both algorithms, the iBTMRG uses a local eigensolver to obtain the Perron vectors. A good initial guess for the eigenvector will significantly improve the performance of the eigensolver. In [5], McCulloch has developed a wave function transformation in iDMRG for the prediction of the wave function as the lattice size is increased. Such a transformation can be effectively translated to our iBTMRG. Therefore, at the end of the first half-period of the process in Fig. 3, the prediction of the next Perron vectors will be

$$
\left\{\begin{array}{l}
\psi_{\text {trial }}^{:}=D_{n+1}^{1 / 2} D_{n}^{-1 / 2} \bar{A}_{n+1}^{:} \bar{\Lambda}_{n+1}=D_{n+1}^{1 / 2} D_{n}^{-1 / 2} \psi: \\
\varphi_{\text {trial }}=D_{n+1}^{1 / 2} D_{n}^{-1 / 2} \bar{B}_{n+1}^{:} \bar{\Gamma}_{n+1}=D_{n+1}^{1 / 2} D_{n}^{-1 / 2} \varphi^{:}
\end{array}\right.
$$

where $D_{n+1}^{1 / 2}$ and $\psi^{:}$are the current canonical center matrix and the current Perron vector. Similar results go to the Perron vectors prediction at the end of the second half-period in 
Fig. 3.

$$
\left\{\begin{array}{l}
\psi_{\text {trial }}^{:}=\bar{\Lambda}_{n+1} \bar{E}_{n+1}^{:} D_{n}^{-1 / 2} D_{n+1}^{1 / 2}=\psi^{:} D_{n}^{-1 / 2} D_{n+1}^{1 / 2} \\
\varphi_{\text {trial }}^{:}=\bar{\Gamma}_{n+1} \bar{F}_{n+1}^{:} D_{n}^{-1 / 2} D_{n+1}^{1 / 2}=\varphi^{:} D_{n}^{-1 / 2} D_{n+1}^{1 / 2}
\end{array}\right.
$$

Note that, for finite fixed $n$, the center matrix $D_{n}^{1 / 2}$ may not be identical for the two halfperiods in Fig. 3 so that the center matrices in Eqs. (10-11) should use their own $D_{n}^{1 / 2}$. When the size of the lattice is small, the $D_{n}^{1 / 2}$ can be replace by $\bar{\Lambda}_{n}$ and $\bar{\Gamma}_{n}$.

At the fixed point of the iBTMRG, the translational invariant iBMPS should bear the canonical form

$$
\left\{\begin{array}{l}
|\psi\rangle=\cdots\left(D_{n-1}^{-1 / 2} \bar{A}_{n}^{\vdots} D_{n}^{1 / 2} \bar{E}_{n}^{:}\right)\left(D_{n-1}^{-1 / 2} \bar{A}_{n}^{:} D_{n}^{1 / 2} \bar{E}_{n}^{:}\right) \cdots \\
|\varphi\rangle=\cdots\left(D_{n-1}^{-1 / 2} \bar{B}_{n}^{:} D_{n}^{1 / 2} \bar{F}_{n}^{:}\right)\left(D_{n-1}^{-1 / 2} \bar{B}_{n}^{:} D_{n}^{1 / 2} \bar{F}_{n}^{:}\right) \cdots
\end{array}\right.
$$

where we take $n$ to be the iteration step when the convergence criterion is met. At the fixed point, the density matrix would be invariant no matter where the bond is located. This leads to the fixed point criterion $\sum_{i} \bar{A}_{n} D_{n} \bar{B}_{n}^{\dagger}=D_{n-1}$. Since $\operatorname{Tr}\left(D_{n}\right)=1$ (with proper normalization $\left\langle\psi^{:} \mid \varphi^{:}\right\rangle=1$ ), one way of measuring the closeness of the two density matrices $D_{n-1}$ and $D_{n}^{\prime}=\sum_{:} \bar{A}_{n}^{:} D_{n} \bar{B}_{n}^{\dagger \dagger}$ is given by the Kullback-Leibler divergence

$$
D_{K L}=\sum_{i} D_{n-1}(i) \log \left(\frac{D_{n-1}(i)}{D_{n}^{\prime}(i)}\right)
$$

In this paper, Eq. (13) is utilized to check the convergence of our iBTMRG.

\subsection{Normalization of the iBMPS}

The fixed point criterion $\sum_{:} \bar{A}_{n}^{:} D_{n} \bar{B}_{n}^{\dagger \dagger}=D_{n-1}$ is equivalent to $\sum_{:}\left(D_{n-1}^{-1 / 2} \bar{A}_{n}^{:} D_{n}^{1 / 2}\right)\left(D_{n-1}^{-1 / 2} \bar{B}_{n}^{:} D_{n}^{1 / 2}\right)^{\dagger}=$ $I$ which means the two matrices $D_{n-1}^{-1 / 2} \bar{A}_{n}^{:} D_{n}^{1 / 2}$ and $D_{n-1}^{-1 / 2} \bar{B}_{n}^{:} D_{n}^{1 / 2}$ satisfy the right-biorthonormal condition. Similarly, $D_{n}^{1 / 2} \bar{E}_{n}^{:} D_{n-1}^{-1 / 2}$ and $D_{n}^{1 / 2} \bar{F}_{n}^{:} D_{n-1}^{-1 / 2}$ must satisfy the left-biorthonormal condition. Thus, the overlap of the iBMPS in Eq. (12) must be exactly $\langle\psi \mid \varphi\rangle=1$ in the fixed point. The normalization $\langle\psi \mid \varphi\rangle=1$ is important when we want to apply the iBMPS to the calculation of expectation value or correlation functions in the thermodynamic limit. However, in practical simulations, the finite number $m$ of states kept by BTMRG and finite iterations can only reach an approximation of the fixed point so that the iBMPS will not be exactly normalized. In [15], Orús and Vidal have developed a method for the orthonormalization of an iMPS in iTEBD, which can also be effectively translated to our iBTMRG. Define two transfer operator $T_{R}$ and $T_{L}$ as shown in Fig. 5 . In order to achieve the rightbiorthonormal condition, assume $\Omega_{R}$ is the right dominant eigenvector of $T_{R}$ and an SVD allows to decompose $\Omega_{R}=U_{R} V_{R}$. We now perform two similarity transformations to the unit cells in Eq. (12) which lead to 


$$
\left\{\begin{aligned}
|\psi\rangle & =\cdots\left(U_{R}^{-1} D_{n-1}^{-1 / 2} \bar{A}_{n}^{:} D_{n}^{1 / 2} \bar{E}_{n}^{:} U_{R}\right)\left(U_{R}^{-1} D_{n-1}^{-1 / 2} \bar{A}_{n}: D_{n}^{1 / 2} \bar{E}_{n}^{:} U_{R}\right) \cdots \\
|\varphi\rangle & =\cdots\left(V_{R}^{-1} D_{n-1}^{-1 / 2} \bar{B}_{n}^{:} D_{n}^{1 / 2} \bar{F}_{n}^{:} V_{R}\right)\left(V_{R}^{-1} D_{n-1}^{-1 / 2} \bar{B}_{n}^{:} D_{n}^{1 / 2} \bar{F}_{n}^{:} V_{R}\right) \cdots
\end{aligned}\right.
$$

Similar arguments apply to $T_{L}$ and its left dominant eigenvector $\Omega_{L}=U_{L} V_{L}$ and lead to the left-biorthonormal iBMPS

$$
\left\{\begin{aligned}
|\psi\rangle & =\cdots\left(U_{L} \bar{A}_{n}^{:} D_{n}^{1 / 2} \bar{E}_{n}^{:} D_{n-1}^{-1 / 2} U_{L}^{-1}\right)\left(U_{L} \bar{A}_{n}^{:} D_{n}^{1 / 2} \bar{E}_{n}^{:} D_{n-1}^{-1 / 2} U_{L}^{-1}\right) \cdots \\
|\varphi\rangle & =\cdots\left(V_{L} \bar{B}_{n}^{:} D_{n}^{1 / 2} \bar{F}_{n}^{:} D_{n-1}^{-1 / 2} V_{L}^{-1}\right)\left(V_{L} \bar{B}_{n}^{:} D_{n}^{1 / 2} \bar{F}_{n}^{:} D_{n-1}^{-1 / 2} V_{L}^{-1}\right) \cdots
\end{aligned}\right.
$$

Accordingly, update the matrices: $\bar{A}_{n}^{:} \leftarrow U_{L} \bar{A}_{n}^{:}, \bar{B}_{n}^{:} \leftarrow V_{L} \bar{B}_{n}^{:}, \bar{E}_{n}^{:} \leftarrow \bar{E}_{n}^{:} U_{R}, \bar{F}_{n}^{:} \leftarrow \bar{F}_{n}^{:} V_{R}$ , and $D_{n-1}^{1 / 2} \leftarrow V_{L} D_{n-1}^{1 / 2} U_{R}$. Then the lattice can be separated into two parts at any bond location where the iBMPS can be expressed as similar to as Eq. (7)

$$
\left\{\begin{array}{l}
|\psi\rangle=\cdots\left(\bar{A}_{n}^{:} D_{n}^{1 / 2} \bar{E}_{n}^{:} D_{n-1}^{-1 / 2}\right) D_{n-1}^{1 / 2}\left(D_{n-1}^{-1 / 2} \bar{A}_{n}^{:} D_{n}^{1 / 2} \bar{E}_{n}^{:}\right) \cdots \\
|\varphi\rangle=\cdots\left(\bar{B}_{n}^{:} D_{n}^{1 / 2} \bar{F}_{n}^{:} D_{n-1}^{-1 / 2}\right) D_{n-1}^{1 / 2}\left(D_{n-1}^{-1 / 2} \bar{B}_{n}^{:} D_{n}^{1 / 2} \bar{F}_{n}^{:}\right) \cdots
\end{array}\right.
$$

\section{Example: 2D classical systems}

In this section, we test our iBTMRG A and iBTMRG $\mathbf{B}$ algorithms on a 2D classical Ising model. The target matrix is $\Pi_{q}^{N}$ where $\Pi_{q}$ is the non-Hermitian fundamental transfer matrix of the general local energy function (LEF)-parameterized Markov random field on an infinitely-long vertical twisted cylindrical lattice with peripheral length $N[10,16]$ (The Ising model is just a special case of a Markov random field). This matrix is intimately related to a 2D Markov additive process and enjoys a very special SVD structure and many fascinating properties [16].

\section{1 iBTMRG in finite systems}

Our iBTMRG algorithms can also be applied to the partition function calculation of classical models in finite lattices. The method to build the reduced biorthonormal bases in iBTMRG $\mathbf{A}$ is essentially the same as the previous BTMRG in [11] which we will refer to as iBTMRG $\mathbf{C}$ for convenience. However, the system-growing strategy of iBTMRG C is different from that of the single-site iBTMRG. Upon the same E•S•E configuration, the previous BTMRG grows the system by adding two sites at each iteration to the system block while keeping the environment block fixed and small. To our knowledge, both proposed single-site iBTMRG algorithms had never been tested before. Here, we will compare the performance of our iBTMRG algorithms and the previous one by computing the free energy of the Ising model on a finite 2D lattice. Figure 6 shows the error of the free energy (i.e., the logarithm of the Perron root of the transfer matrix $\left.\Pi_{q}\right)$ of an anisotropic Ising model $\left(J_{x}=-J_{y}\right.$ where $J_{x}$ and $J_{y}$ represent the interaction between horizontally and vertically neighboring spins) at the critical temperature $T_{c}=2 / \log (1+\sqrt{2})$ for system size $N=160$ and various number 
of states $m$ kept by iBTMRG. We also compare the performance of the finite-size variant of BTMRG (fBTMRG) for the three algorithms where the Perron states from the iBTMRG were used as the initial BMPS states and were further optimized variationally. From Fig. 6, we can see that iBTMRG A and B exhibit almost identical performance which were further slightly improved by their variational finite-size variants. For fBTMRGs, algorithms $\mathrm{A}$ and $\mathrm{B}$ give rise to very close results and only outperform algorithm $\mathrm{C}$ a bit. Although iBTMRG $\mathbf{C}$ has the merit of high efficiency, it is interesting to note that its accuracy is far worse and remain constant for all values of $m$. This is because, in the system-growing stage, the size of the environment block is kept fixed and small so that the entanglements between the two subsystems remain fixed and small irrespective of the reduced basis size $m$.

\section{2 iBTMRG in the thermodynamic limit}

In iBTMRG, finding the Perron states by an iterative eigensolver is the most time-consuming part so that the overlap between the initial wave function and the variational optimum $\left\langle\psi_{\text {trial }}^{:} \mid \psi_{\text {opt }}^{:}\right\rangle$will dominate the performance of an iBTMRG algorithm. Figure 7 shows the fidelity $1-\left\langle\psi_{\text {trial }}^{:} \mid \psi_{\text {opt }}^{:}\right\rangle$(see McCulloch [5]) between the Perron states predictors and the optimal ones for the above anisotropic Ising model at criticality with $m=40$ states kept in the reduced basis. Before the peripheral size of the system grows larger than $N=50$, the center matrices in Eqs. (10-11) must be replaced by the un-canonized center matrix $\bar{\Lambda}_{n}$ and $\bar{\Gamma}_{n}$ since the density matrices cannot be diagonalized to real positive eigenvalues until $N=50$. From Fig. 7, the un-canonized center matrices seem inappropriate for the usage of wave function transformation. Once the canonical center matrices are utilized, the fidelity quickly drops down to $10^{-6}$ and continues to decay in a nearly constant rate. Moreover, both predictions for algorithms A and B have very close effectiveness and the right Perron state transformation appears to prevail with respect to the left Perron state transformation. Another important issue concerns the convergence of the translationally invariant fixed point of the iBMPS. In this paper, we use the Kullback-Leibler divergence to monitor the convergence of the algorithm. The inset of Fig. 7 shows the convergence to the fixed point of the iBMPS with respect to the number of iterations. In view of the degeneracy of the Perron root and the long-range spin correlation at the criticality, the convergence appears to be quite slow. However, in off-critical regions, the convergence can be as fast as reaching $10^{-12}$ as the size exceeding $N=200$.

Once we have the translationally invariant iBMPS (after normalization $\langle\psi \mid \varphi\rangle=1$ ), the calculation of the expectation value or the two-point correlator in the thermodynamic limit can be very efficient. For illustration, we take an isotropic $\left(J_{x}=J_{y}=J\right)$ Ising model as an example and calculate its magnetization as a function of the temperature and its spin-spin correlation function at the critical temperature. Now consider Eq. (16), if we free the unit cells $\psi_{\alpha, \xi}^{\sigma_{L} \sigma_{L}^{\prime}, \sigma_{R} \sigma_{R}^{\prime}} \equiv D_{n-1}^{1 / 2}\left(D_{n-1}^{-1 / 2} \bar{A}_{n}^{\sigma_{L} \sigma_{R}} D_{n}^{1 / 2} \bar{E}_{n}^{\sigma_{L}^{\prime} \sigma_{R}^{\prime}}\right)$ and $\varphi_{\beta, \zeta}^{\sigma_{L} \sigma_{L}^{\prime}, \sigma_{R} \sigma_{R}^{\prime}} \equiv$ 
$D_{n-1}^{1 / 2}\left(D_{n-1}^{-1 / 2} \bar{B}_{n}^{\sigma_{L} \sigma_{R}} D_{n}^{1 / 2} \bar{F}_{n}^{\sigma_{L}^{\prime} \sigma_{R}^{\prime}}\right)$ then we have Perron states in the thermodynamic limit expressed as $\psi_{\alpha, \xi}^{\sigma_{L} \sigma_{L}^{\prime}, \sigma_{R} \sigma_{R}^{\prime}}$ and $\varphi_{\beta, \zeta}^{\sigma_{L} \sigma_{L}^{\prime}, \sigma_{R} \sigma_{R}^{\prime}}$ with respect to a dual biorthonormal bases. Note that the spins $\sigma_{L} \sigma_{L}^{\prime}$ and $\sigma_{R} \sigma_{R}^{\prime}$ are two neighboring spins pairs located on the left and right side of the system block, and the spins $\sigma_{L} \sigma_{R}$ and $\sigma_{L}^{\prime} \sigma_{R}^{\prime}$ are two (infinitely) far distant aligned spins. Therefore, the magnetization in the thermodynamic limit can be calculated by

$$
\left\langle\sigma_{R}\right\rangle=\sum_{\sigma_{R}} \sigma_{R} \sum_{\alpha=\beta, \xi=\zeta \sigma_{L} \sigma_{L}^{\prime} \sigma_{R}^{\prime}} \psi_{\alpha, \xi}^{\sigma_{L} \sigma_{L}^{\prime}, \sigma_{R} \sigma_{R}^{\prime}} \varphi_{\beta, \zeta}^{\sigma_{L} \sigma_{L}^{\prime}, \sigma_{R} \sigma_{R}^{\prime}}
$$

For an infinite $2 \mathrm{D}$ isotropic Ising model, the exact solution for the magnetization is $\left(1-\left(\sinh \left(2 J / k_{B} T\right)\right)^{-4}\right)^{1 / 8}$ [17]. Here, we compute the magnetization by Eq. (17) and the exact formula as in Fig. 8 where the inset shows the relative error between the numerical result and the exact one. Similarly, the spin-spin correlation between two infinitely far distant spins in the thermodynamic limit can be calculated by

$$
\left\langle\sigma_{L} \sigma_{R}\right\rangle=\sum_{\sigma_{L} \sigma_{R}} \sigma_{L} \sigma_{R} \sum_{\alpha=\beta, \xi=\zeta \sigma_{L}^{\prime} \sigma_{R}^{\prime}} \psi_{\alpha, \xi}^{\sigma_{L} \sigma_{L}^{\prime}, \sigma_{R} \sigma_{R}^{\prime}} \varphi_{\beta, \zeta}^{\sigma_{L} \sigma_{L}^{\prime}, \sigma_{R} \sigma_{R}^{\prime}}
$$

At the critical temperature, the exact spin-spin correlation function scales as $G(r) \propto$ $r^{-1 / 4}\left(1+O\left(r^{-2}\right)\right)$ [18]. Ideally, Eq. (18) will be zero but in practice it can only be seen as the correlation of two spins separated by a distance $r$ equal to half of the system size $N$ where the fixed point criterion is met. This implies that we can regard Eq. (18) as the correlation function in the thermodynamic limit as $r=N / 2$. Figure 9 shows the scaling behavior of the spin-spin correlator compared with the exact solution. It is worthy of noting that, in the low-temperature region nearby the criticality, our iBTMRG behaves quite prone to getting stuck. Fortunately, by introducing White's correction [14] to the reduced biorthonormalized bases, the algorithm exhibits much improved efficiency and convergence with the correction weight around $10^{-3}-10^{-4}$. In [15], Orús and Vidal have conducted the same calculation as in Figs. 8-9 by using the iTEBD algorithm for the same Ising model (see Figs. 11-12 in [15]). For the magnetization, although the iTEBD has achieved a better accuracy than $\mathrm{BBTMRG}$, they are not on the same conditions. In addition to the main difference already mentioned in the introduction, another difference is that the transfer matrix they have dealt with is Hermitian which is much numerically well-conditioned than the non-Hermitian case. More importantly, for the two-point correlation calculation, instead of evaluating a very long tensor network as in [15], we use a very efficient formula Eq. (18) and obtain a result that is comparable with respect to the iTEBD. 


\section{Conclusions}

In this paper, we give a thorough Biorthonormal Matrix-Product-State (BMPS) analysis of the Transfer-Matrix Renormalization-Group (TMRG) for non-Hermitian matrices and propose a "single-site" infinite-size Biorthonormal TMRG (iBTMRG) algorithm to directly obtain a dual infinite-BMPS (iBMPS) which are translationally invariant in the thermodynamic limit. Different from the standard DMRG that the MPS was established on a series of reduced orthonormal bases, the BMPS are built on a dual series of reduced biorthonormal bases for the left and right Perron states of a non-Hermitian matrix. We propose two alternative methods for the construction of the dual biorthonormal bases and compare their numerical performance in both finite and infinite systems. We also develop an efficient wave function transformation of the iBTMRG, an analogy of McCulloch [5] in the infinite-DMRG, to predict the wave function as the lattice size is increased. The resulting translationally invariant iBMPS not only allows for investigating bulk properties of a strongly correlated system in the thermodynamic limit without the boundary effect but also allows for the evaluation of expectation and two-point correlation function of the system very efficiently. For illustration, we calculate the magnetization as a function of the temperature and the critical spin-spin correlation function in the thermodynamic limit for a $2 \mathrm{D}$ classical Ising model.

\section{References}

[1] S. R. White, Phys. Rev. Lett. 69, 2863 (1992); I. Peschel, X. Wang, M. Kaulke and K. Hallberg (eds.) Density Matrix Renormalization, Lecture Notes in Physics (Springer, Berlin, 1999); U. Schollwöck, Rev. Mod. Phys. 77, 259-3152005 (2005); K. Hallberg, Adv. Phys. 55, 477 (2006)

[2] G. Vidal, Phys. Rev. Lett. 91, 147902 (2003); G. Vidal, Phys. Rev. Lett. 93, 040502 (2004)

[3] J. Dukelsky et al., Europhys. Lett. 43, 457 (1998); T. Nishino et al., Int. J. Mod. Phys.

B 13, 1 (1999); F. Verstraete, D. Porras, and J. I. Cirac, Phys. Rev. Lett. 93, 227205 (2004); F. Verstraete, V. Murg, and J. I. Cirac, Adv. Phys. 57, 143 (2008)

[4] G. Vidal, Phys. Rev. Lett. 98, 070201 (2007)

[5] I. P. McCulloch, arXiv:0804.2509 (2008)

[6] T. Nishino, J. Phys. Soc. Jpn. 64, 3598 (1995); T. Nishino and K. Okunishi, J. Phys. Soc. Jpn. 66, 3040 (1997)

[7] R. J. Bursill, T. Xiang, and G. A. Gehring, J. Phys.: Condens. Matter 8, L583 (1996); X. Wang and T. Xiang, Phys. Rev. B 56, 5061 (1997); N. Shibata, J. Phys. A 36, 381 (2003); A. E. Feiguin and S. R. White, Phys. Rev. B 72, 220401 (2005)

[8] A. Kemper, A. Schadschneider and J. Zittartz, J. Phys. A: Math. Gen. 34, L279 (2001);

T. Enss and U. Schollwöck, J. Phys. A: Math. Gen. 34, 7769 (2001)

[9] Y. Hieida, J. Phys. Soc. Jpn. 67, 369 (1998); E. Carlon, M. Henkel and U. Schollwöck, 
Phys. Rev. E 63, 036101 (2001); S. Trebst et al., Phys. Rev. Lett. 96, 250402 (2006); M. Cramer et al., Phys. Rev. Lett. 101, 063001 (2008); T. Barthel et al., Phys. Rev. A 79, 053627 (2009)

[10] Y.-K. Huang and S.-N. Yu, Physica A 390, 801 (2011)

[11] Y.-K. Huang, Phys. Rev. E 83, 036702 (2011)

[12] S. Östlund and S. Rommer, Phys. Rev. Lett. 75, 3537 (1995)

[13] M. A. Martín-Delgado and G. Sierra, Int. J. Mod. Phys. A 11, 3145 (1996); S. Rommer and S. Östlund, Phys. Rev. B 55, 2164 (1997); I. P. McCulloch, J. Stat. Mech.: Theor. Exp. P10014 (2007); J. I. Cirac and F. Verstraete, J. Phys. A: Math. Theor. 42, 504004 (2009); U. Schollwöck, Ann. Phys. 326, 96 (2011)

[14] S. R. White, Phys. Rev. B 72, 180403 (2005)

[15] R. Orús and G. Vidal, Phys. Rev. B 78, 155117 (2008)

[16] Y.-K. Huang and S.-N. Yu, Physica A 389, 736 (2010)

[17] L. Onsager, Phys. Rev. 65,117 (1944)

[18] H. Au-Yang and J. H. H. Perk, Phys. Lett. A 104, 131 (1984) 

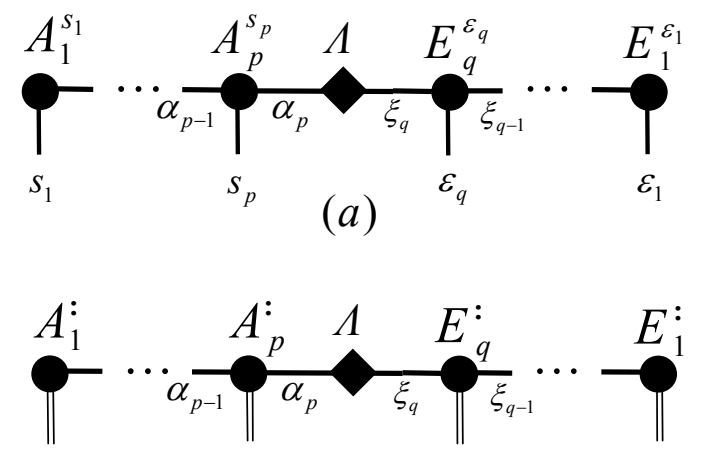

(b)

FIG. 1. (a) The tensor network representation of the MPS in the standard $\mathrm{S} \bullet \mathrm{E}$ configuration. (b) The tensor network representation of the MPS in our special $\mathrm{E} \bullet \mathrm{S} \bullet \mathrm{E}$ configuration. 


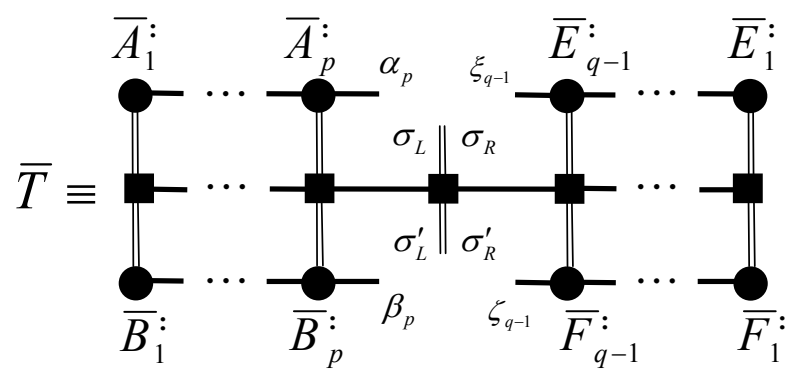

FIG. 2. The tensor network representation of the reduced transfer matrix $\bar{T}$ associated with the dual BMPS of BTMRG upon the $\mathrm{E} \bullet \mathrm{S} \bullet \mathrm{E}$ configuration. 


$$
\begin{gathered}
\cdots-\bar{A}_{n}:-\bar{\Lambda}_{n}-\bar{E}_{n}: \cdots \\
\cdots-\frac{\|}{B}:-\bar{\Gamma}_{n}-\bar{F}_{n}:-\cdots
\end{gathered}
$$

Step 1. $\ \begin{aligned} & \text { Adding two sites to the system block } \\ & \text { and obtain the Perron states }\end{aligned}$

$$
\begin{gathered}
\cdots-\bar{A}_{n}:-\psi^{:}-\bar{E}_{n}^{:}-\cdots \\
\| \\
\cdots-\frac{\|}{\|_{n}}-\varphi: \varphi^{:}-\bar{F}_{n}^{:}-\cdots
\end{gathered}
$$

$$
\begin{aligned}
& \text { Step 2. \erform SVD } \\
& \cdots-\bar{A}:-A_{n+1}^{:}-\Lambda_{n+1}-\bar{E}_{n}^{:}-\cdots \\
& \begin{array}{c}
\| \\
\|-\frac{\|}{B}:-B_{n+1}^{:}-\Gamma_{n+1}-\bar{F}_{n}^{:}-\cdots
\end{array} \\
& \cdots-\bar{A}_{\|}:-\bar{A}_{n}: \bar{\Lambda}_{n+1}-\bar{E}_{\|}^{:}-\cdots \\
& \cdots-\frac{\|}{B}{ }_{n}-\frac{\|}{B_{n+1}}:-\bar{\Gamma}_{n+1}-\bar{F}_{n}^{:}-\cdots
\end{aligned}
$$

$$
\begin{aligned}
& \text { Step 4. } \ \text { Canonize the BMPS } \\
& \cdots-\bar{A}_{n}^{:}-\bar{A}_{n+1}^{\prime:}-\sqrt{D}_{n+1}-\bar{E}_{n}^{\prime:}-\cdots \\
& \cdots-\bar{B}_{n}:-\bar{B}_{n+1}^{\prime:}-\sqrt{D_{n+1}} \bar{F}_{n}^{\prime:}-\cdots
\end{aligned}
$$

Repeat Step 1-4. Adding two sites to the environment block

FIG. 3. The main steps of iBTMRG A, which grows the lattice by adding first two sites to the system block and then adding two sites to the environment block, constitute a period of the algorithm with respect to a repeated fragment of the final iBMPS. 


$$
\begin{aligned}
& \text { Step } 2.1 \downarrow \text { Restore the bases } \\
& \cdots-A_{\|}^{:}-P_{n} \psi^{:} Q_{n}-E^{:}-\cdots \\
& \cdots-\stackrel{\|}{B}_{n}:-W_{n} \varphi: R_{n}-\stackrel{\|}{F}:-\cdots
\end{aligned}
$$

$$
\begin{aligned}
& \text { Step } 2.2 \downarrow \text { Perform SVD } \\
& \cdots-\|_{n}^{:}-A_{n+1}^{:}-\Lambda_{n+1}-\mathbb{\|}_{n}^{:}-\cdots \\
& \cdots-\left\|_{n}^{:}-\right\|_{n+1}^{:}-\Gamma_{n+1}-\stackrel{\|}{F}_{n}^{:}-\cdots
\end{aligned}
$$$$
\begin{aligned}
& \text { Step } 3.1 \downarrow \text { Change the bases } \\
& \cdots-\bar{A}_{n}^{:}-P_{n}^{-1} A_{n+1}^{:}-\bar{\Lambda}_{n+1}-\bar{E}_{n}^{:}-\cdots \\
& \cdots-\frac{\|}{B}{ }_{n}^{:}-W_{n}^{-1} B_{n+1}^{:}-\bar{\Gamma}_{n+1}-\bar{F}_{n}:-\cdots
\end{aligned}
$$

Step $3.2 \downarrow$ Perform Biorthonormalization

$$
\begin{aligned}
& \cdots-\bar{A}:-\bar{A}_{n+1}^{:}-\bar{A}_{n+1}^{\prime}-\bar{E}_{n}^{:}-\cdots \\
& \cdots-\frac{\|}{B}:-\frac{\|}{B_{n+1}}: \bar{\Gamma}_{n+1}^{\prime}-\bar{F}_{n}^{:}-\cdots
\end{aligned}
$$

FIG. 4. The main steps of IBTMRG B is the same as IBTMRG A only with the steps 2-3 enclosed in a rectangle in Fig. 3 being replaced by the routine depicted in this figure. 


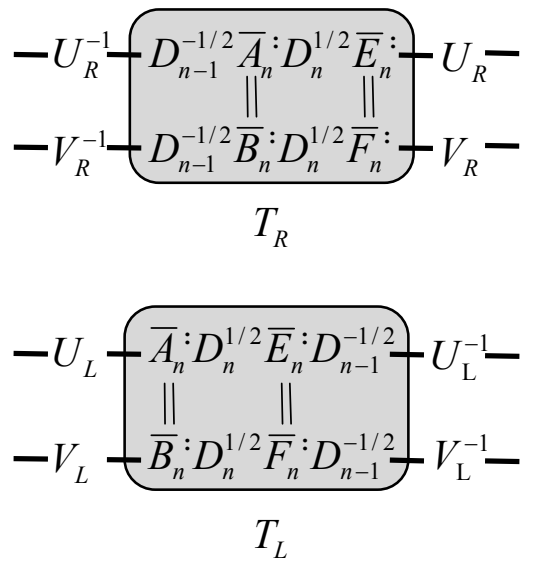

FIG. 5. To achieve the right (left)-biorthonormalization condition, we must perform two similarity transformations to the unit cells of the iBMPS where $\Omega_{R}=U_{R} V_{R}\left(\Omega_{L}=U_{L} V_{L}\right)$ is the right (left) dominant eigenmatrix of the transfer operator $T_{R}\left(T_{L}\right)$. 


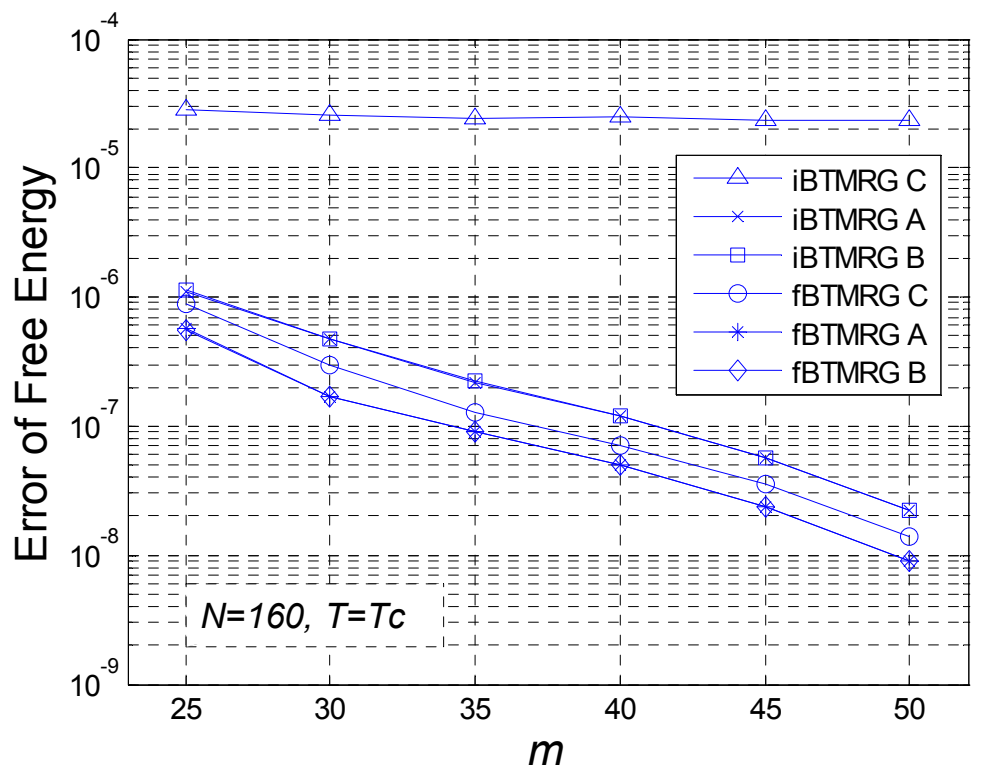

FIG. 6. The comparison of the infinite-size (iBTMRG) and finite-size (fBTMRG) variants of three BTMRG algorithms by plotting the error of the free energy of an anisotropic Ising model with lattice size $N=160$ at the critical temperature $T_{c}=2 / \log (1+\sqrt{2})$. 


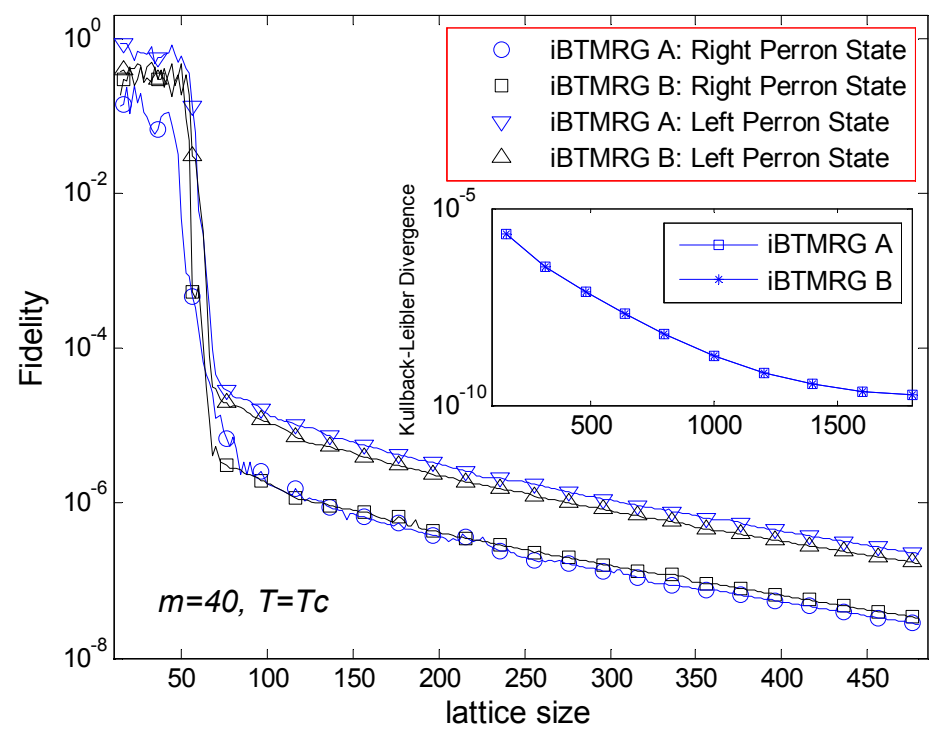

FIG. 7. The fidelity $1-\left\langle\psi_{\text {trial }}^{:} \mid \psi_{\text {opt }}^{:}\right\rangle$between the Perron states predictors and the optimal ones for the anisotropic Ising model at criticality with $m=40$ states kept in the reduced basis. The inset shows the convergence of the Kullback-Leibler divergence to the fixed point of the iBMPS with respect to the number of iterations. 


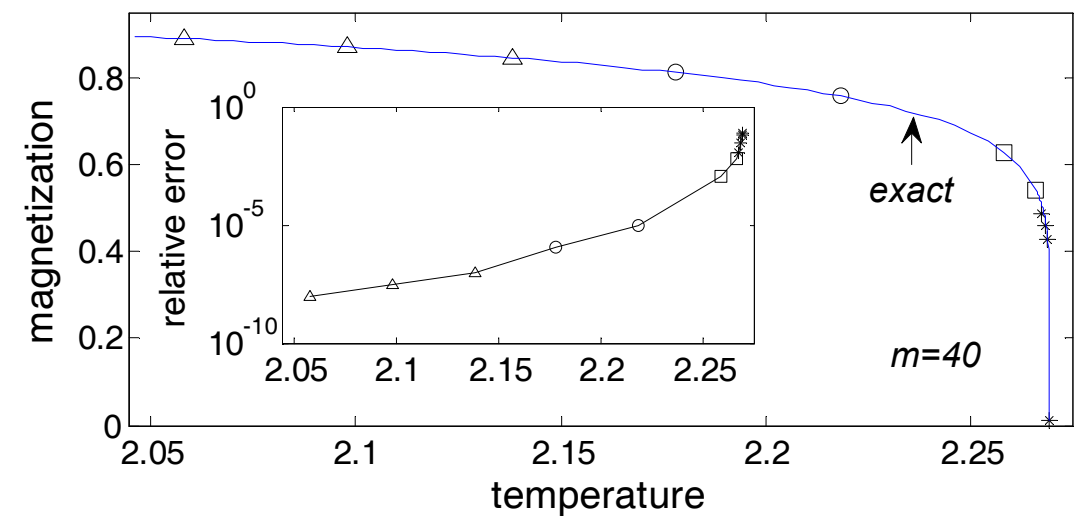

FIG. 8. The magnetization as a function of the temperature of an isotropic Ising model in the thermodynamic limit with $m=40$. The inset shows the relative error of the magnetization with respect to the exact solution. 


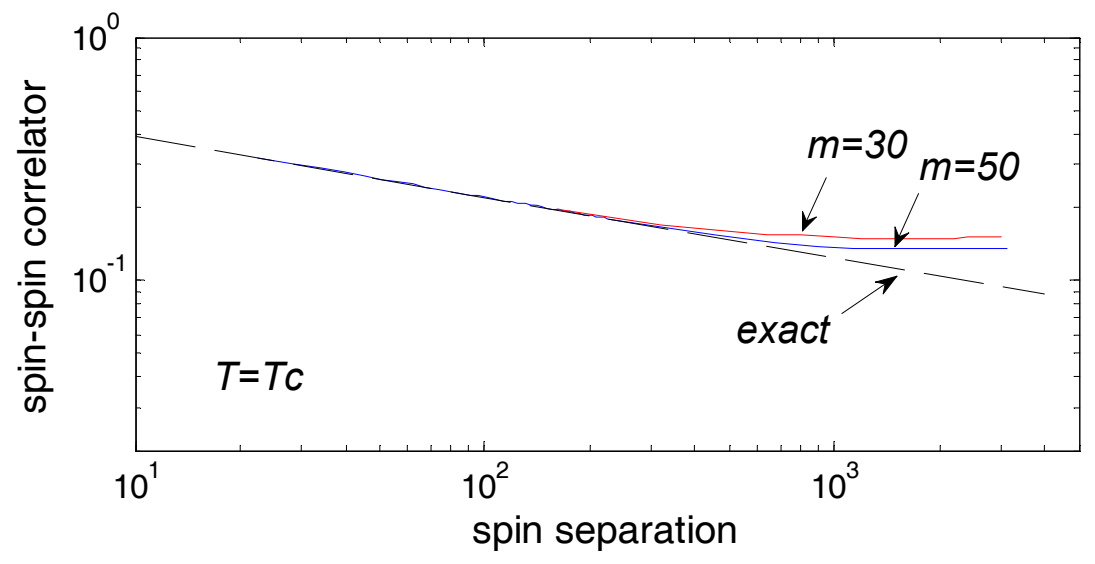

FIG. 9. The scaling behavior of the spin-spin correlator of an isotropic Ising model at the critical temperature obtained from Eq. (18) which is compared with the exact solution. 